\title{
LINAC RF STRUCTURES FOR THE SPALLATION NEUTRON SOURCE*
}

\author{
J. H. Billen, H. Takeda, and T. S. Bhatia \\ Los Alamos National Laboratory, Los Alamos, NM 87545 USA
}

\section{Abstract}

The Spallation Neutron Source (SNS) project is a collaboration among Argonne, Brookhaven, Lawrence Berkeley, Los Alamos, and Oak Ridge National Laboratories. Los Alamos is responsible for the linac that accelerates the $\mathrm{H}^{-}$beam from $2.5 \mathrm{MeV}$ to $1 \mathrm{GeV}$. For the baseline design, scheduled for completion in 2005, the linac will deliver to the accumulator ring a beam of 1.1MW average power. In the SNS linac, a conventional 402.5-MHz drift-tube linac (DTL) accelerates the beam from 2.5 to $20 \mathrm{MeV}$, at which point $805-\mathrm{MHz}$ structures take over. The $805-\mathrm{MHz}$ linac consists of a coupledcavity drift-tube linac (CCDTL), followed by a coupledcavity linac (CCL). The DTL uses permanent magnet quadrupoles inside the drift tubes arranged in a FOFODODO lattice; the focusing period is $4 \beta \lambda$ long at 402.5 MHz. The CCDTL and CCL use electromagnetic quadrupole magnets external to the rf structure; the FODO lattice period is $12 \beta \lambda$ long at $805 \mathrm{MHz}$. The cavity field profile maintains smooth longitudinal focusing strength per unit length. High cavity stored energy reduces the effect of beam chopping on the cavity fields. The bore radius is $1.25 \mathrm{~cm}$ in the DTL, and increases in the CCDTL and CCL in several steps consistent with adequate shunt impedance, to a final value of $2.0 \mathrm{~cm}$. The rf structures are compatible with a future upgrade to a beam power of 4.4 MW.

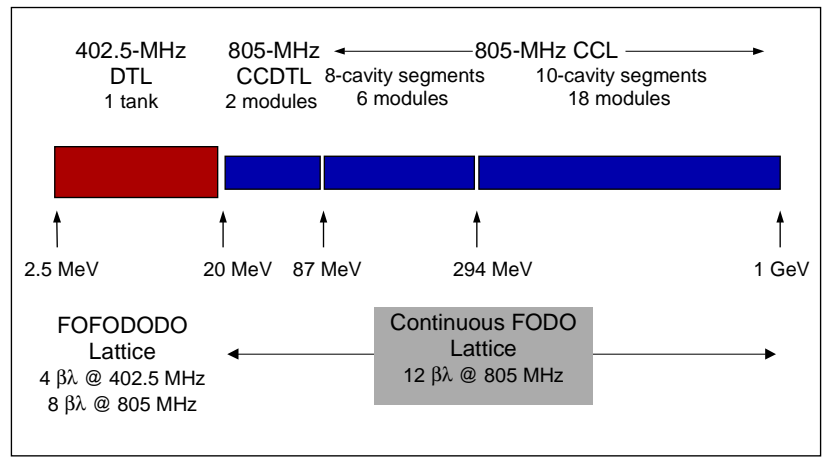

Figure 1. SNS linac layout for $1 \mathrm{MW}$ beam power.

\section{INTRODUCTION}

Figure 1 shows the major items to be delivered by Los Alamos: the $402.5-\mathrm{MHz}$ drift-tube linac and the $805-\mathrm{MHz}$ coupled-cavity structures. These are room-temperature copper accelerating structures. During operation, the water coolant temperature will control the resonant frequency. Other SNS components appear in papers at this conference. Figure 2 is a plot of the cavity shunt

*Work supported by the Office of Energy Research, Basic Energy Science of the US Department of Energy, and by Oak Ridge National Laboratory. impedance $\mathrm{ZT}^{2}$ as a function of particle velocity, which is affected strongly by the choice of bore radius. Values of $\mathrm{ZT}^{2}$ in the figure include all expected losses in the cavities. The SNS linac requires high cavity stored energy in order to reduce the effect of beam chopping on the cavity fields. When designing such cavities, one is usually limited either by the maximum power densities that can be cooled or by the tendency of the cavity to spark at high electric fields. With an rf duty factor of $6.7 \%$, the power density is not the limitation, though water cooling is required. Figure 3 plots the peak surface electric field relative to the Kilpatrick field as a function of particle velocity. The Kilpatrick field is $19.47 \mathrm{MV} / \mathrm{m}$ at 402.5 $\mathrm{MHz}$ and $26.06 \mathrm{MV} / \mathrm{m}$ at $805 \mathrm{MHz}$. Values below 1.5 Kilpatrick represent a conservative design.

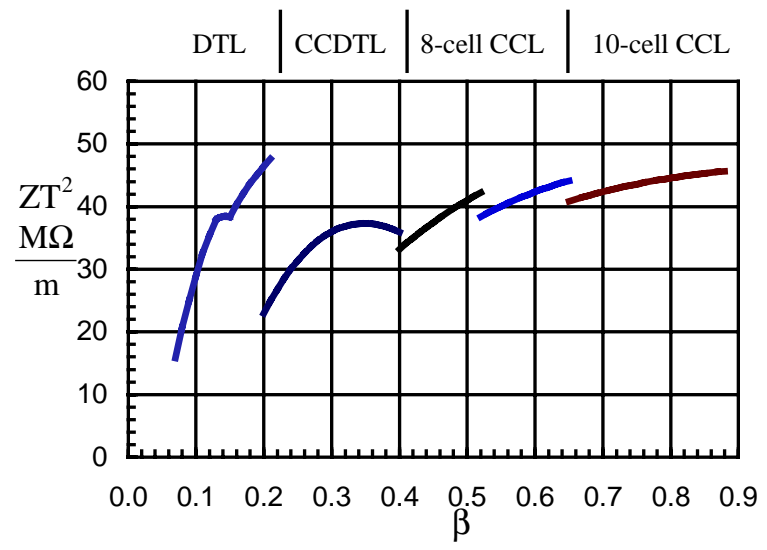

Figure 2. Cavity shunt impedance versus particle velocity.

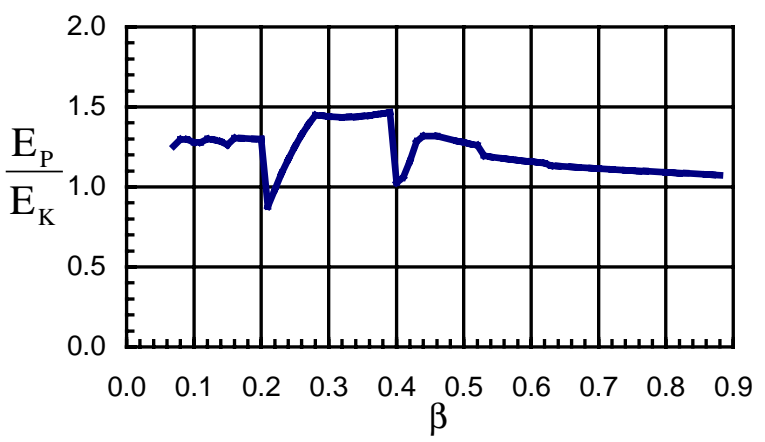

Figure 3. Peak surface electric field in terms of the Kilpatrick field versus particle velocity.

\section{DRIFT-TUBE LINAC}

Table 1 lists the DTL design parameters. The DTL has a single tank with 84 accelerating gaps. The contour plots in Figure 4 show three representative cells computed by SUPERFISH [1] The lower left corner of each figure of revolution is the center of the gap. The tank diameter D will be slightly larger than $43 \mathrm{~cm}$ and the drift-tube 
diameter $\mathrm{d}=9 \mathrm{~cm}$. The space for post couplers $(\mathrm{D}-\mathrm{d}) / 2$ is about $91 \%$ of $\lambda / 4$ at $402.5 \mathrm{MHz}$, an ideal distance for ensuring good stability. A series of 12 static slug tuners along the bottom of the tank will provide several $\mathrm{MHz}$ of tuning capability.

Drift tubes have the full $9-\mathrm{cm}$ diameter over a long enough length to house the permanent-magnet quadrupoles. We adjust the face geometry for high shunt impedance constant peak surface electric field of $\sim 1.3$ Kilpatrick for an average axial electric field $\mathrm{E}_{0}=3.0$ $\mathrm{MV} / \mathrm{m}$. We vary the drift-tube length to tune each cell to 402.5 MHz. Several parameters change with $\beta$ over part of the DTL. The bore radius increases from $1.25 \mathrm{~cm}$ to 1.5 $\mathrm{cm}$ over about 11 cells between $\beta=0.13$ and $\beta=0.15$. The face angle varies from 3 degrees to 65 degrees (see Figure 4). The detailed nose shape includes an inner radius $R_{\mathrm{i}}$ in the range $0.16 \mathrm{~cm}$ to $0.35 \mathrm{~cm}$, an outer radius $\mathrm{R}_{\mathrm{o}} \approx 3 \mathrm{R}_{\mathrm{i}}$, and a short flat segment between them. This flat segment (in the range $0.05 \mathrm{~cm}$ to $0.4 \mathrm{~cm}$ ) reduces the peak surface electric field at the expense of a small reduction in shunt impedance.

Two values appear in Table 1 for the beam current and total rf power. The lower number is for the baseline 1MW beam power design and the larger number is for an upgrade to $4 \mathrm{MW}$ discussed below. The power figures include an expected $20 \%$ reduction in the SUPERFISHcalculated $\mathrm{ZT}^{2}$.

Table 1. DTL Design Summary

\begin{tabular}{|l|l|}
\hline Frequency & $402.5 \mathrm{MHz}$ \\
\hline Injection/Final Energy & $2.5 / 20.0 \mathrm{MeV}$ \\
\hline Micropulse Current (1MW/4MW) & $28 / 56 \mathrm{~mA}$ \\
\hline Macropulse Current (1MW/4MW) & $18 / 36 \mathrm{~mA}$ \\
\hline Average Accelerating Field & $3.0 \mathrm{MV} / \mathrm{m}$ \\
\hline Quadrupole Focusing Lattice & FOFODODO \\
\hline Peak Surface Electric Field & $1.3 \mathrm{Kilpatrick}$ \\
\hline Length & $8.26 \mathrm{~m}$ \\
\hline Radial Aperture & $1.25 \mathrm{and} 1.5 \mathrm{~cm}$ \\
\hline Number of Cells & 84 \\
\hline Number of Post Couplers & 41 \\
\hline Total RF Power (1MW/4MW) & $1.6 / 1.9 \mathrm{MW}$ \\
\hline
\end{tabular}

\section{COUPLED-CAVITY STRUCTURES}

After the DTL at 20-MeV beam energy, the linac uses $805-\mathrm{MHz}$ coupled-cavity structures composed of relatively short contiguous sections of a few accelerating cavities called segments. The accelerating cavities within a segment are connected to short, side-mounted coupling cavities in the usual way. Longer side coupling cavities span the drift spaces between segments. The magnetic coupling between cavities is 5\%. A transverse focusing period consists of two segments and two drift spaces that contain electromagnetic quadrupole (EMQ) singlets. Diagnostic elements also occupy some of the drift spaces. Table 2 lists the major linac parameters.

In SNS there are three structure sections characterized by the type and number of cavities per segment. In the first part, from $20 \mathrm{MeV}$ to about $87 \mathrm{MeV}$, a segment contains two CCDTL cavities of total length $3 \beta \lambda$. The
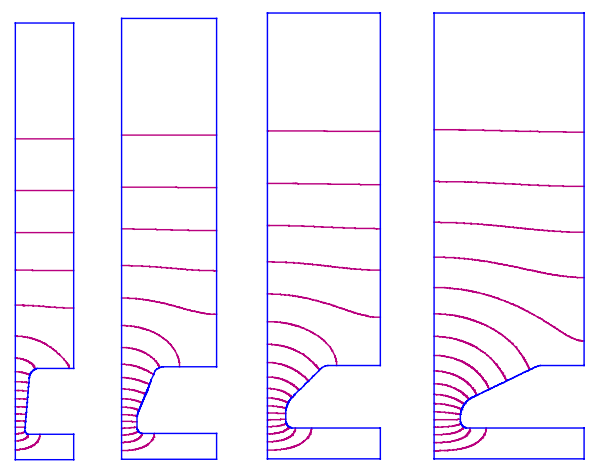

Figure 4. DTL cells for $\beta=0.08,0.13,0.15$, and 0.20.

drift spaces between segments are also $3 \beta \lambda$. Segments in the second part to $294 \mathrm{MeV}$ contain $8 \mathrm{CCL}$ cavities of total length $4 \beta \lambda$, but the drift spaces are now $2 \beta \lambda$ long. The major part of the linac has 10-cavity CCL segments of total length $5 \beta \lambda$ and $1 \beta \lambda$ drift spaces. The design has several mechanical, rf, and beam-dynamics advantages:

- There are no transitions in the transverse focusing lattice, which has a constant period of $12 \beta \lambda$ at 805 MHz.

- The EMQs are accessible and can be aligned independently from the rf structures.

- Furnace-brazed rf modules eliminate most mechanical rf and vacuum joints.

- The structure contains no bridge couplers.

Table 2. CCDTL and CCL Design Summary

\begin{tabular}{|l|l|}
\hline Frequency & $805 \mathrm{MHz}$ \\
\hline Injection/Final Energy & $20 / 1000 \mathrm{MeV}$ \\
\hline Micropulse Current (1MW/4MW) & $56 / 112 \mathrm{~mA}$ \\
\hline Macropulse Current (1MW/4MW) & $18 / 72 \mathrm{~mA}$ \\
\hline Average Accelerating Field & $3.7 \mathrm{MV} / \mathrm{m}$ \\
\hline Energy Gain (real-estate average) & $2.14 \mathrm{MeV} / \mathrm{m}$ \\
\hline Quadrupole Focusing Lattice & FODO \\
\hline Transverse Focusing Period & $12 \beta \lambda$ \\
\hline Length & $458 \mathrm{~m}$ \\
\hline Radial Aperture & 1.5 to $2.0 \mathrm{~cm}$ \\
\hline Peak Surface Electric Field & $<1.5 \mathrm{Kilpatrick}$ \\
\hline Structure Power & $80 \mathrm{MW}$ \\
\hline Beam Power (1MW/4MW) & $1.1 / 4.4 \mathrm{MW}$ \\
\hline Number of RF Modules & 26 \\
\hline Number of Accelerating Cavities & 2560 \\
\hline Number of Segments & 347 \\
\hline Klystrons/Module (1MW/4MW) & $2 / 3$ \\
\hline
\end{tabular}

\subsection{Coupled-Cavity Drift-Tube Linac}

The first two rf modules have 61 and 30 CCDTL [2] segments. The bore radius $R_{b}=1.5 \mathrm{~cm}$. Each segment contains two cavities like the one in Figure 5. The CDTFISH code from Ref. 1 helps automate the design and optimization process. At each end of a range of particle velocities $\beta$, we first optimize the gap g, drift-tube diameter $\mathrm{d}$, drift-tube face angle $\alpha_{\mathrm{f}}$, and equator flat length $\mathrm{F}_{\text {eq }}$. The optimum has high $\mathrm{ZT}^{2}$ and is within the acceptable range of power density and peak surface 
electric field. We adjust the cavity diameter $\mathrm{D}$ for resonance at $805 \mathrm{MHz}$. If a parameter changes by a substantial amount from its initially chosen value, we repeat the optimization of the parameters studied earlier. After finishing the optimization studies, we design a cavity for every 0.01 step in $\beta$, again varying $D$ for resonance at $805 \mathrm{MHz}$, using linear interpolation for $\mathrm{g}$, d, $\alpha_{\mathrm{f}}$, and $\mathrm{F}_{\text {eq }}$. between the two end-point values. Beam dynamics studies use the transit-time factor data for these representative cavities.

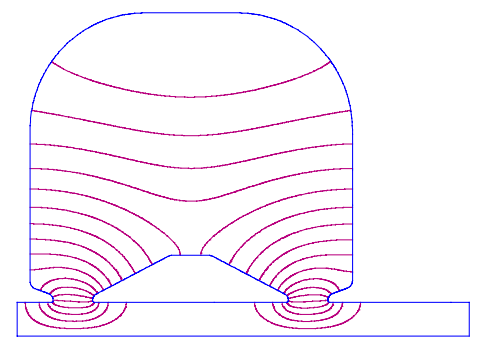

Figure 5. CCDTL cavity for $\beta=0.24$.

\subsection{Coupled-Cavity Linac}

Above $\beta \sim 0.4$ the conventional CCL shown in Figure 6 is more efficient than the CCDTL. The exact energy of the transition depends upon other details of the $\mathrm{rf}$ partitioning. The first $6 \mathrm{rf}$ modules in the CCL have 8 cavities per segment. At $\beta=0.65$ a space of length $1 \beta \lambda$ is long enough for the EMQ, so later modules have 10 cavities per segment. The CCL has three sections of different bore radius. The smallest radius $R_{b}=1.5 \mathrm{~cm}$ occurs at the low $\beta$ end (modules 3 and 4 ) and matches $R_{b}$ at the end of the CCDTL. We increase $R_{b}$ to $1.75 \mathrm{~cm}$ in modules $5,6,7$, and 8 and to $2.0 \mathrm{~cm}$ in modules 9 through 26 . The effects of these changes on $\mathrm{ZT}^{2}$ can be seen in Figure 2. For each bore radius we follow an optimization procedure like that described for the CCDTL. The parameters varied for high $\mathrm{ZT}^{2}$ are the gap, the cone angle of the nose, and the inner and outer radii of the cavity wall. Again, the cavity diameter varies for tuning to $805 \mathrm{MHz}$.

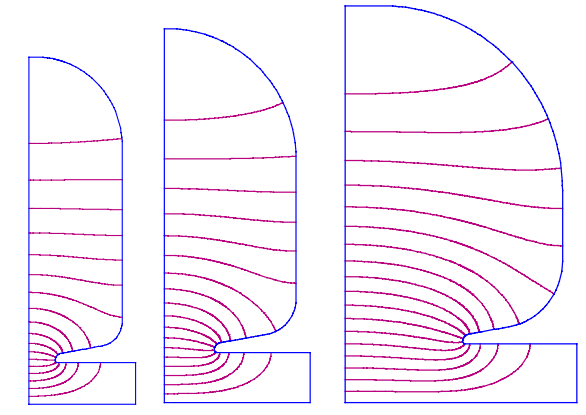

Figure 6. CCL cells for $\beta=0.43,0.60$, and 0.87 .

\subsection{RF Partitioning}

The coupled-cavity linac structures will operate in the $\pi / 2$ mode, which has remarkably good field stability, low power-flow droop, and negligible power-flow phase shift for a few percent coupling. These structures must be divided into a relatively small number of $\mathrm{rf}$ modules whose power requirements are matched to the available power supplies. We plan to use commercial $805-\mathrm{MHz}$ klystrons with a rated output power of $2.5 \mathrm{MW}$ at the SNS duty factor. Of this power, $2.0 \mathrm{MW}$ is available for supplying the cavity fields and accelerating beam. With 300 or fewer cavities (including coupling cells) and 5\% coupling between cavities, the mode density near the $\pi / 2$ operating mode remains low enough for easy tuning of the structures.

In addition to these power requirements and the limit on the total number of cavities, we have imposed a few other requirements on the partitioning. For thermal management, we allow only one structure type in an $\mathrm{rf}$ module. For example, the CCDTL ends at the end of module 2 and the 8-cavity CCL ends at the end of module 7. Also, bore radius changes occur only at ends of modules. Finally, a module can only be lengthened or shortened by adding or subtracting an entire segment. A CCDTL segment has 4 cavities and a CCL segment has either 16 or 20 cavities. The resulting partitioning scheme has 26 rf modules, with each module driven by two klystrons.

\section{UPGRADING TO 4 MW}

The initial installation of the SNS linac will deliver a 1.1MW beam to the accumulator ring. One design requirement is the ability to upgrade to four times the initial beam power. The proposed solution is to duplicate the entire $20-\mathrm{MeV}, 402.5-\mathrm{MHz}$ front end, double the beam current in each leg, and funnel the two beams together before the beam enters the $805-\mathrm{MHz}$ linac.

Each rf module in the $805-\mathrm{MHz}$ linac has 3 drive irises, placed at the $1 / 6,3 / 6$, and $5 / 6$ power points, which is optimum for 4-MW operation. The 1-MW linac, which has lower beam loading, uses the two outside irises. The only major shutdown required is for installation of the duplicate front end and the beam funnel. Upgrading the CCDTL and CCL can be done by installing klystrons during scheduled down times with no impact on the running schedule.

\section{CONCLUSION}

We have outlined the design of the SNS linac. The design has no transitions in the focusing lattice after the $20-\mathrm{MeV}$ DTL. Magnets are not susceptible to drift-tube vibrations because they mount outside the rf structure where they remain accessible and serviceable. Power densities are manageable and surface electric fields are conservative.

\section{REFERENCES}

1 J. H. Billen and L. M. Young, POISSON SUPERFISH, Los Alamos National Laboratory report LA-UR-1834 (revised November 14, 1998).

2 J. H. Billen, F. L. Krawczyk, R. L. Wood, and L. M. Young, "A New RF Structure for Intermediate-Velocity Particles," Proceedings of the 1994 International Linac Conference, Vol. 1, p. 341 (August 21-26, 1994). 